\title{
CheMinX: A Next Generation XRD/XRF for Quantitative Mineralogy and Geochemistry on Mars
}

Elizabeth Rampe ${ }^{1}$, David Blake ${ }^{2}$, Philippe Sarrazin ${ }^{3}$, Thomas Bristow ${ }^{2}$, Marc Gailhanou ${ }^{4}$, Barbara Lafuente ${ }^{2}$, Valerie Tu ${ }^{5}$, Kris Zacny ${ }^{6}$, and Robert Downs ${ }^{7}$

${ }^{1}$ Astromaterials Research and Exploration Science Division, NASA Johnson Space Center, Houston, TX, USA (elizabeth.b.rampe @ nasa.gov; 281-483-0216)

${ }^{2}$ Exobiology Branch, NASA Ames Research Center, Moffett Field, CA, USA

${ }^{3}$ ExaminArt, LLC, Mountain View, CA USA

${ }^{4}$ Aix Marseille Universite, CNRS, IM2NP, Marseille, France

${ }^{5}$ Jacobs/JETS at NASA Johnson Space Center, Houston, TX, USA

${ }^{6}$ Honeybee Robotics, Pasadena, CA, USA

${ }^{7}$ Department of Geosciences, University of Arizona, Tucson, AZ USA 


\section{Introduction}

X-ray diffraction (XRD) and X-ray fluorescence (XRF) analyses provide the most diagnostic and complete mineralogical and geochemical characterization of rocks and soils by any spacecraft-capable technique, improved upon only by sample return and analyses in terrestrial laboratories [1]. In a mineralogically complex sample such as a basalt or in aqueously altered basaltic sediments, XRD can definitively identify and quantify all primary and secondary minerals, establish their individual elemental compositions, and quantify the X-ray amorphous component. When coupled with XRF, the composition of the amorphous component can be calculated and trace element substitutions within igneous minerals (e.g., Ti in olivine) can be discriminated. XRD paired with quantitative XRF analyses of drilled rock and loose soil samples on any planetary surface will yield:

- Identification of all minerals present $>1 \mathrm{wt} . \%$.

- Quantification of all minerals >3 wt. \%, including their structure states and cation occupancies.

- Bulk geochemistry with ppm detection limits of some elements.

- Abundance of all major elements present in each mineral ( $\mathrm{H}$ and above).

- Valence state of all elements, including speciation of multi-valent species, such as Fe.

- Abundance and composition of X-ray amorphous components, if present.

Other mineralogical and geochemical instruments have been (or will soon be) deployed on Mars orbiters and landers, including visible-near-infrared (vis-NIR) and thermal-infrared spectrometers, X-ray fluorescence and particle-induced X-ray emission spectrometers, Mössbauer spectrometers, Raman spectrometers, laser-induced breakdown spectrometers, and gas chromatograph mass spectrometers. The mineralogical datasets returned from orbiters like the Mars Reconnaissance Orbiter and Mars Global Surveyor and from landers like the Mars Exploration Rovers (MER) have demonstrated that the martian surface is compositionally and mineralogically heterogeneous. Indeed, we now know that aqueous environments (requisite for life and a key indicator of habitability) on early Mars varied over space and time [2-6]. However, only XRD coupled with quantitative XRF, can return the quantitative mineralogy and mineral chemistry necessary to fully characterize geologic history and habitability.

\section{The MSL-CheMin Instrument}

The first X-ray diffractometer flown in space is the Chemistry and Mineralogy (CheMin) instrument on the Mars Science Laboratory Curiosity rover (Figure 1; [7]). CheMin is a combination XRD/XRF and operates in transmission mode. A collimated Co X-ray beam with a diameter of $\sim 70 \mu \mathrm{m}$ is transmitted through a powdered sample, and the position and energy of the transmitted X-rays are recorded by a CCD. Downlinked two-dimensional XRD patterns are transformed to 1-dimensional XRD patterns and processed using commercially available data reduction programs. Although MSL-CheMin's CCD detects both diffracted and fluoresced X-ray photons from the sample, the capability to quantify XRF data was descoped prior to flight with the result that XRF data from MSL-CheMin are only qualitative. For bulk geochemistry, MSLCheMin relies on data collected by the Alpha Particle X-ray Spectrometer (APXS) collected from 
drill tailings or samples dumped from the Sample Acquisition, Sample Processing and Handling (SA/SPaH)-Collection and Handling for In-Situ Martian Rock Analysis (CHIMRA) instrument [8]. Diagenesis in Gale crater, however, has caused compositional heterogeneities at the $\mathrm{mm}$ to $\mathrm{cm}$ scale [9-11] such that the chemical composition of the sample analyzed by APXS may not be representative of that analyzed by CheMin.
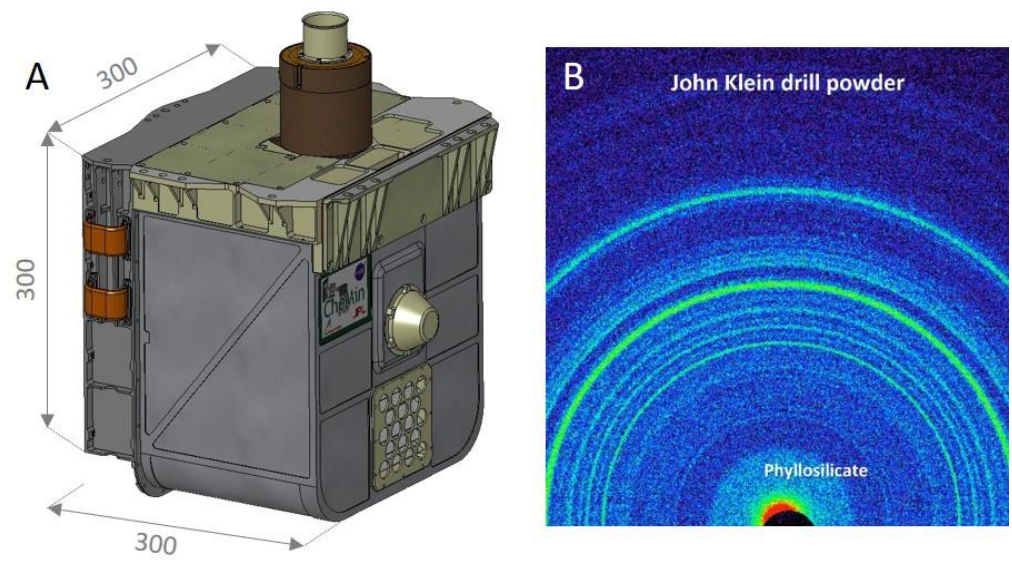

Figure 1. (A) The MSL-
CheMin instrument, with
dimensions (in mm), mass is
$10.5 \mathrm{~kg}$. (B) An example of $2 \mathrm{D}$
XRD data from the "John
Klein" sample, drilled from the
first habitable environment
identified by Curiosity. Pattern
resolution is $\sim 0.35^{\circ} 2 \theta$.

During nominal operations, MSL-CheMin accepted scooped soil or drilled rock sieved to $<150 \mu \mathrm{m}$ and portioned to $40-50 \mathrm{mg}$ by SA/SPaH-CHIMRA. An anomaly in the drill feed mechanism on sol 1536 prohibited nominal operations of SA/SPaH-CHIMRA such that MSLCheMin now accepts powdered rock samples directly from the drill bit [12]. Samples are delivered to one of 27 disc-shaped, reusable sample cells in a wheel configuration. Cells are $8 \mathrm{~mm}$ in diameter and $175 \mu \mathrm{m}$ thick with X-ray transparent Mylar or Kapton windows. Piezoelectric actuators on each cell pair induce convective grain motion during analysis such that sample powder in the cell flows through the X-ray beam in random orientations during the course of an analysis. Samples are typically analyzed for a total of 22 hours, split over three different nights. Each night of analysis costs the rover $\sim 500 \mathrm{~W} \cdot \mathrm{hrs}$ of energy and $\sim 200 \mathrm{MiBit}$ of data volume.

The Curiosity rover has been studying a sequence of $\sim 3.5 \mathrm{Ga}$ old fluvio-lacustrine and eolian deposits in Gale crater since landing in August 2012. CheMin analyses of 26 drilled rock samples and three modern eolian samples have transformed our view of Mars' geologic history and habitability. CheMin's mineralogical data were used by the Curiosity science team to identify habitable environments throughout the traverse [13-14], constrain $\mathrm{P}_{\mathrm{CO} 2}$ in the early Hesperian [15], and evaluate discrete changes in aqueous environments over time [16-17]. Orbital vis-NIR data of Gale crater suggested that smectite clay minerals were present only in specific strata [18-19]. CheMin's ground truth mineralogy, however, demonstrated that smectite clays are present in significant amounts throughout most of the lacustrine Murray formation (comprising hundreds of meters of strata over many kilometers). Furthermore, changes in the XRD patterns indicate that the smectite structure gradually evolves from trioctahedral to dioctahedral in younger strata, suggesting a change from closed to open system alteration in situ or in the catchment [16]. Some discoveries by MSL-CheMin remain enigmatic. Tridymite and/or cristobalite in a few samples may be an indication of regional silicic volcanism or may result from a style of diagenesis unknown in the terrestrial environment [20-21]. X-ray amorphous material is present in all samples analyzed to date, from ancient fluvio-lacustrine to ancient and modern eolian deposits [12], but its formational process is not understood. The amount and elemental composition of the amorphous materials can be calculated using CheMin bulk mineralogy and APXS bulk chemistry. The 
calculated compositions are inconsistent with volcanic or impact glass, suggesting that at least some of the amorphous component formed from water-rock interactions (and surprisingly, persisted for $>3.5$ Gyrs).

MSL-CheMin operations over the past eight years have highlighted some issues that must be addressed for XRD on future Mars rovers:

- CheMin's design utilizes reusable sample cells; however, in some cases, cells cannot be emptied after analysis.

- CheMin requires XRF data from a companion instrument on the rover (APXS) to determine bulk chemistry of the samples.

- CheMin's size, mass, and power restrict its use to Flagship-class missions.

Deployment of XRD/XRF on smaller (i.e., MER-class) rovers requires further miniaturization, and the availability of simpler sample collection capabilities than were implemented for the MSL mission.

\section{CheMinX Design}

CheMinX, a next-generation XRD/XRF instrument, is based on similar principles as MSLCheMin, but benefits from a decade of advancements in geometry design and subsystem miniaturization [22]. The XRD measurement of CheMinX is similar to MSL-CheMin, but uses different components and a different layout to optimize its geometry. Diffracted photons are collected by a CCD in direct illumination, critical for energy-selective detection of XRD photons in Mars' radiation environment. Whereas MSL-CheMin uses APXS bulk sample compositions, CheMinX uses an internal Silicon Drift Detector (SDD) to provide a concurrent XRF measurement of the sample. CheMinX sample cells are redesigned for a more compact and lower cost sample handling subsystem. A fixed tuning fork is combined with multiple single-use cells in a cartridge/dispenser arrangement to address the issue of clogged sample cells experienced with MSL-CheMin. A preliminary mechanical design of CheMinX is shown in Figure 2.
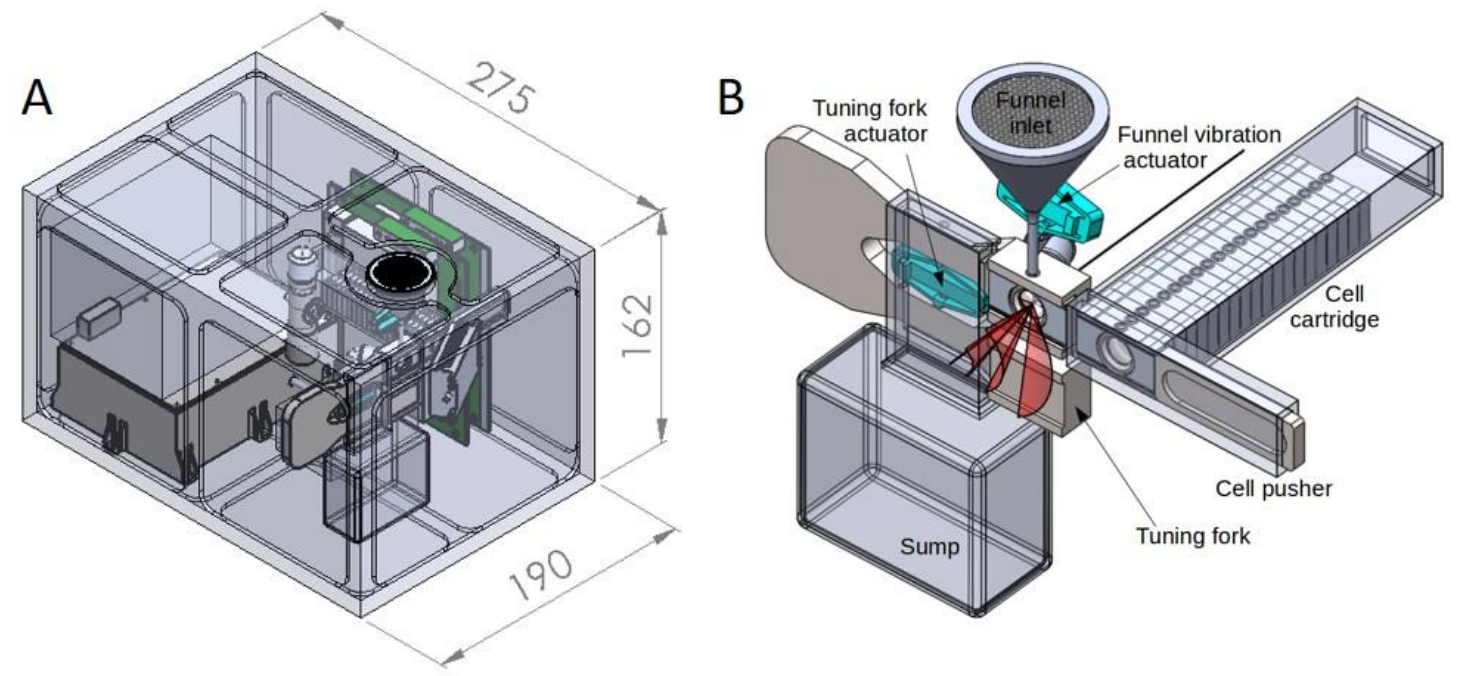

Figure 2. Preliminary mechanical design of the CheMinX flight instrument. (A) Instrument with dimensions (in $\mathrm{mm}$ ), projected mass is $5 \mathrm{~kg}$. (B) Sample handling subsystem for the vibrated sample method based on single-use cells in a cartridge dispenser system. This sampling subsystem is being developed as part of a NASA PICASSO19 grant awarded to P. Sarrazin. 
The CheMinX XRD geometry is based on an architecture demonstrated in hundreds of commercial XRD instruments. Terra, a commercial spin-off of MSL-CheMin and marketed by Olympus Corp. (Figure 3), is a field-portable XRD that has been used by scientists in field sites across the world [23]. This geometry resulted from a ray-tracing study of MSL-CheMin geometries with high aspect ratio (1024 X 256 pixel) detectors. It was found that reduced surface area CCD detectors can be used with no loss in throughput, angular resolution, or angular range. The loss in detector coverage is compensated for by an optimized collimator design. Placement of the CCD at a $30^{\circ}$ tilt from the direct beam enables an increased sample-to-detector distance that provides an improvement in $2 \theta$ resolution. In its Terra-like implementation, CheMinX will provide a resolution of $0.3^{\circ} 2 \theta \mathrm{FWHM}$, slightly improved over MSL-CheMin's $0.35^{\circ} 2 \theta$. This geometry modification enables the use of vis-NIR spectroscopy CCD detectors in place of the custom X-ray CCD of MSL-CheMin. The cost of these detectors as well as their power requirement for deep cooling are dramatically reduced compared to MSL-CheMin.
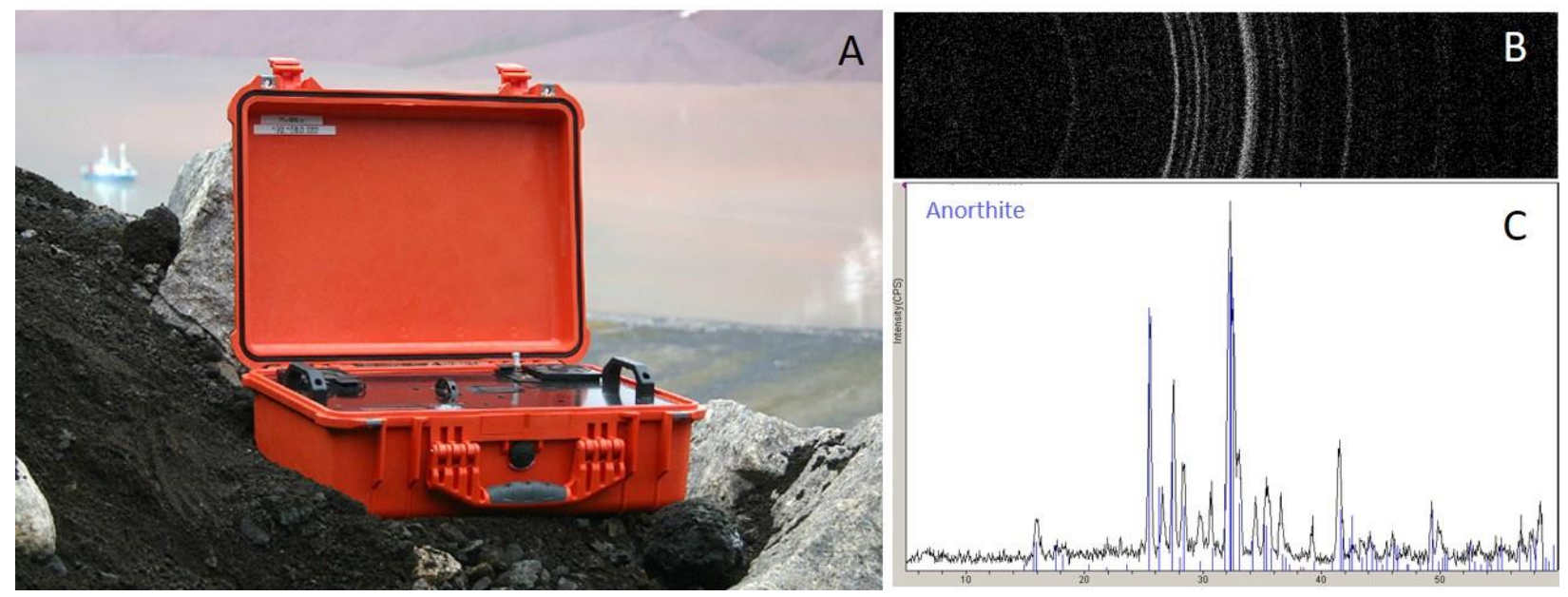

Figure 3. (A) Terra commercial portable XRD instrument, prototype deployed in Svalbard during 2007 AMASE field deployment. Mass: $14.5 \mathrm{~kg}$ (including case, batteries and embedded computer), power: $75 \mathrm{~W}$, typical analysis time: $15 \mathrm{~min}$ to $1 \mathrm{hr}$. (B) $2 \mathrm{D}$ image, and (C) 1D diffraction pattern from Terra of a sample of anorthite. CheMinX will use the same detector and XRD geometry as Terra, but we are investigating hybrid-pixel technology in place of CCDs, as well.

Elemental data are obtained from XRF spectra collected by an SDD in reflection geometry. These spectra are quantified using Fundamental Parameters (FP) calculations. Depending on the background, trace elements can be detected down to a few 10s of ppm, given sufficient collection time.

CheMinX has the capability to measure the direct beam intensity at Co Ka. This enables the measurement of sample absorption, which varies with chemistry, compactness, and thickness. Absorption inside the sample affects the overall diffracted intensity and the shape of the diffraction peaks, and is as such important for accurately modeling the diffraction pattern and evaluating bulk mineralogy and crystal size and strain. The direct beam intensity measurement is obtained passively with a solid polycrystalline material (e.g., diamond, silicon) positioned in the beam-stop structure to diffract a single partially masked ring, proportional to the transmitted beam intensity, on a lesser populated region of the CCD. 
Rapid and cost-effective development of flight instruments requires the availability of mature technologies for critical components. High TRL subsystems have been developed in collaboration with industrial partners: Specialized full-frame and frame-transfer X-ray CCD detectors (Teledyne e2v); custom FPGA-based electronics for low-noise CCD operation and embedded data processing (Baja Technology); miniature microfocused X-ray tubes (ROENTGEN-TECHNIK, RTW); and compact high-voltage power supplies (Battel Engineering). These components will find applications in CheMinX as well as other planetary XRD and XRF instruments [24-26].

\section{Sample Collection and Delivery}

CheMinX requires the collection and delivery of powdered rock materials or soils. The development of simplified Sample Acquisition and Delivery Systems (SA/DS) is critical for the deployment of XRD instruments on smaller rovers. Such systems are being developed for deployment on MER-class rovers [22]. As part of a PICASSO19 grant awarded to P. Sarrazin, we are fabricating and testing simpler sample processing and delivery systems, including a microsampling drill designed for low power and load requirements, and will demonstrate CheMinX with powdering drills and arm prototypes developed at Honeybee Robotics.

As a result of an anomaly of the drill feed mechanism on MSL, drill powders are now directly delivered to the CheMin instrument without sieving, by positioned the drill over the CheMin inlet and rotating the drill bit backwards. As-received drill powders are sufficiently fine to produce high-quality XRD patterns, demonstrating that a complex sample sieving and portioning system is not necessary for sample delivery to CheMinX on future martian rovers.

\section{The Utility of CheMinX on Future Mars Rovers}

Substantial reductions in mass, volume, and power relative to MSL-CheMin are now possible for planetary XRD/XRF instruments while achieving improved resolution and 10X reduction in data collection time. These future instruments will benefits from improved XRF performance through the use of a dedicated SDD placed in backscattering geometry. CheMinX will provide quantitative mineralogy and elemental chemistry from drilled rocks and scooped soils on Mars in a package suitable for deployment on a MER-class rover.

Quantitative mineralogy combined with bulk chemistry provide the most useful in situ dataset for the characterization of Mars' geologic history and habitability. There are presently only two localities on Mars for which we will have these data: (1) Gale crater and the rocks and soils analyzed by instruments on Curiosity and (2) Jezero crater and the rocks and soils that will be collected by Perseverance (but only if samples are returned). Data collected by orbiters, landers, and rovers (in particular, MSL-CheMin data from Curiosity's traverse) have revealed a striking diversity in both composition and mineralogy on all scales of observation $-\mathrm{cm}$ to tens of kilometers. Quantitative mineralogical data from two locations on Mars (both ancient crater lakes) are not sufficient to characterize the igneous and aqueous histories of early Mars and its habitability. It is crucial to send multiple MER-class rovers with XRD/XRF capabilities to different locations on the martian surface to fully characterize the geologic history of the planet.

Furthermore, prior to humans landing on the martian surface, it will be crucial to determine bulk mineralogy and geochemistry to evaluate the presence of in situ resources (e.g., hydrated minerals) and potential toxins (e.g., perchlorates). Deployment of CheMinX instruments on 
landers or rovers designed to scout potential human landing sites will facilitate the selection of resource-rich terrain and will allow mission engineers and scientists to better prepare for human occupation of Mars.

\section{References}

[1]. Velbel, M. A. (2018). Crystallography on Mars: Curiosity's bragging right. American Mineralogist, 103(6), 837-838, https://doi.org/10.2138/am-2018-6468CCBYNCND.

[2]. Squyres, S. W., and Knoll, A. H. (2005). Sedimentary rocks at Meridiani Planum: Origin, diagenesis, and implications for life on Mars. Earth and Planetary Science Letters, 240(1), 1-10, https://doi.org/10.1016/j.eps1.2005.09.038.

[3]. Rogers, A. D., and Christensen, P. R. (2007). Surface mineralogy of Martian low-albedo regions from MGS-TES data: Implications for upper crustal evolution and surface alteration. Journal of Geophysical Research: Planets, 112(E1), https://doi.org/10.1029/2006JE002727.

[4]. Morris, R. V., Klingelhöfer, G., and Bell, J. F. (2008). Iron mineralogy and aqueous alteration on Mars from the MER Mössbauer spectrometers. The Martian Surface: Composition, mineralogy, and physical properties. Cambridge University Press, UK, 339365.

[5]. Mustard, J. F., Murchie, S. L., Pelkey, et al. (2008). Hydrated silicate minerals on Mars observed by the Mars Reconnaissance Orbiter CRISM instrument. Nature, 454, 305-309, https://doi.org/10.1038/nature07097.

[6]. Ehlmann, B. L., and Edwards, C. S. (2014). Mineralogy of the martian surface. Annual Review of Earth and Planetary Sciences, 42, 291-315, https://doi.org/10.1146/annurev-earth060313-055024.

[7]. Blake, D., Vaniman, D., Achilles, C., et al. (2012). Characterization and calibration of the CheMin mineralogical instrument on Mars Science Laboratory. Space Science Reviews, 170, 341-399, https://doi.org/10.1007/s11214-012-9905-1.

[8]. Anderson, R. C., Jandura, L., Okon, A. B., et al. (2012). Collecting samples in Gale crater, Mars; an overview of the Mars Science Laboratory Sample Acquisition, Sample Processing and Handling System. Space Science Reviews, 170, 57-75, https://doi.org/10.1007/s11214012-9898-9.

[9]. Berger, J. A., Schmidt, M. E., Gellert, R., et al. (2017). Zinc and germanium in the sedimentary rocks of Gale Crater on Mars indicate hydrothermal enrichment followed by diagenetic fractionation. Journal of Geophysical Research: Planets, 112(8), 1747-1772, https://doi.org/10.1002/2017JE005290.

[10]. Nachon, M., Mangold, N., Forni, O., et al. (2017). Chemistry of diagenetic features analyzed by ChemCam at Pahrump Hills, Gale crater, Mars. Icarus, 281, 121-136, https://doi.org/10.1016/j.icarus.2016.08.026.

[11]. L'Haridon, J., Mangold, N., Fraeman, A. A., et al. (submitted). Iron mobility during diagenesis as observed by ChemCam at the Vera Rubin ridge, Gale crater, Mars. Journal of Geophysical Research: Planets.

[12]. Rampe, E. B., Blake, D. F., Bristow, T. F., et al. (2020). Mineralogy and geochemistry of sedimentary rocks and eolian sediments in Gale crater, Mars: A review after six Earth years 
of exploration with Curiosity. Geochemistry, 80(2), 125605, https://doi.org/10.1016/j.chemer.2020.125605.

[13]. Grotzinger, J. P., Sumner, D. Y., Kah, L. C., et al. (2014). A habitable fluvio-lacustrine environment at Yellowknife Bay, Gale crater, Mars. Science, 343, 6169, http://dx.doi.org/10.1126/science.1242777.

[14]. Grotzinger, J. P., Gupta, S., Malin, M. C., et al. (2015). Deposition, exhumation, and paleoclimate of an ancient lake deposit, Gale crater, Mars. Science, 350(6257), aac7575, https://doi.org/10.1126/science.aac7575.

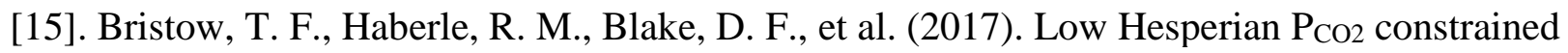
from in situ mineralogical analysis at Gale Crater, Mars. Proceedings of the National Academy of Sciences, 114(9), 2166-2170, https://doi.org/10.1073/pnas.1616649114.

[16]. Bristow, T. F., Rampe, E. B., Achilles, C. N., et al. (2018). Clay mineral diversity and abundance in sedimentary rocks of Gale crater, Mars. Science Advances, 4(6), eaar3330, https://doi.org/10.1126/sciadv.aar3330.

[17]. Rampe, E. B., Ming, D. W., Blake, D. F., et al. (2017). Mineralogy of an ancient lacustrine mudstone succession from the Murray formation, Gale crater, Mars. Earth and Planetary Science Letters, 471, 172-185, http://dx.doi.org/10.1016/j.epsl.2017.04.021

[18]. Milliken, R. E., Grotzinger, J. P., and Thomson, B. J. (2010). Paleoclimate of Mars as captured by the stratigraphic record in Gale Crater. Geophysical Research Letters, 37(4), https://doi.org/10.1029/2009GL041870.

[19]. Fraeman, A. A., Ehlmann, B. L., Arvidson, R. E., et al. (2016). The stratigraphy and evolution of lower Mount Sharp from spectral, morphological, and thermophysical data sets. Journal of Geophysical Research: Planets, 121, 1713-1736, https://doi.org/10.1002/2016JE005095.

[20]. Morris, R. V., Vaniman, D. T., Blake, D. F., et al. (2016). Silicic volcanism on Mars evidenced by tridymite in high- $\mathrm{SiO}_{2}$ sedimentary rock at Gale crater. Proceedings of the National Academy of Sciences, 113(26), 7071-7076, https://doi.org/10.1073/pnas.1607098113.

[21]. Yen, A. S., Morris, R. V., Ming, D. W., et al. (submitted). High-temperature hydrothermal history at Gale crater, Mars. Journal of Geophysical Research: Planets.

[22]. Sarrazin, P., Bristow, T. F., Blake, D. F., et al. (2019). "CheMinX: A Next Generation XRD/XRF for Mars Exploration.” LPS L, abstract 2239.

[23]. Sarrazin, P., Brunner, W., Blake, D., et al. (2008). Field studies of Mars analog materials using a portable XRD/XRF instrument. LPS XXXIX, abstract 2421.

[24]. Walroth, R. C., Blake, D. F., Sarrazin, P., et al. (2018). MAPX-PIXE: A fill field micro Xray fluorescence imager for astrobiology applications on ocean worlds. LPS XLIX, abstract 2233.

[25]. Blake, D. F., Bristow, T. F., Chen, J., et al. (2019a). XTRA: An extraterrestrial regolith analyzer for lunar soil. LPS L, abstract 1144.

[26]. Blake, D. F., Sarrazin, P., Bristow, T. F., et al. (2019b). CheMin-V: A definitive mineralogy instrument for the Venera-D mission. LPS L, abstract 1468. 